\title{
Tweet, Tweet, Retweet: Conversational Aspects of Retweeting on Twitter
}

\author{
danah boyd \\ Microsoft Research \\ dmb@microsoft.com / @zephoria
}

\author{
Scott Golder \\ Cornell / Microsoft Research \\ sag262@cornell.edu / @redlog
}

\author{
Gilad Lotan \\ Microsoft \\ gilad1@microsoft.com / @gilgul
}

\begin{abstract}
Twitter-a microblogging service that enables users to post messages ("tweets") of up to 140 characters-supports a variety of communicative practices; participants use Twitter to converse with individuals, groups, and the public at large, so when conversations emerge, they are often experienced by broader audiences than just the interlocutors. This paper examines the practice of retweeting as a way by which participants can be "in a conversation." While retweeting has become a convention inside Twitter, participants retweet using different styles and for diverse reasons. We highlight how authorship, attribution, and communicative fidelity are negotiated in diverse ways. Using a series of case studies and empirical data, this paper maps out retweeting as a conversational practice.
\end{abstract}

\section{Introduction}

Social media has enabled conversations to occur asynchronously and beyond geographic constraints, but they are still typically bounded by a reasonably welldefined group of participants in some sort of shared social context. Network-driven genres (e.g., social network sites, microblogging) complicate this because people follow the conversations in the context of individuals, not topical threads. Yet, conversations still emerge between dyads and among groups.

On Twitter, a popular microblogging service, directed conversations usually involve use of the “@user" syntax to refer to others and address messages to them; Honeycutt and Herring [9] examine this conversational practice. A subset of participants also uses hashtags (\#'s) to mark tweets topically so that others can follow conversations centering on a particular topic. However, a third convention, known as retweeting, has yet to be analyzed.

Structurally, retweeting is the Twitter-equivalent of email forwarding where users post messages originally posted by others. This convention has no uniform grammar, although 'RT@user msg' is one common form. Because Twitter limits tweets to 140 characters, messages being retweeted must frequently be modified to accommodate the additional notation meant to indicate that the message is a retweet.

While retweeting can simply be seen as the act of copying and rebroadcasting, the practice contributes to a conversational ecology in which conversations are composed of a public interplay of voices that give rise to an emotional sense of shared conversational context. For this reason, some of the most visible Twitter participants retweet others and look to be retweeted. This includes users of all kinds, but notably marketers, celebrities and politicians.

Because Twitter's structure disperses conversation throughout a network of interconnected actors rather than constraining conversation within bounded spaces or groups, many people may talk about a particular topic at once, such that others have a sense of being surrounded by a conversation, despite perhaps not being an active contributor. The stream of messages provided by Twitter allows individuals to be peripherally aware without directly participating.

Retweeting brings new people into a particular thread, inviting them to engage without directly addressing them. In this article, we argue that, as with link-based blogging [13], retweeting can be understood both as a form of information diffusion and as a means of participating in a diffuse conversation. Spreading tweets is not simply to get messages out to new audiences, but also to validate and engage with others.

The goal of this paper is to describe and map out the various conventions of retweeting, and to provide a framework for examining retweeting practices. This serves multiple purposes. First, as Twitter and other technologies begin providing features to support practices like retweeting, it is crucial to understand the diversity of behaviors taking place. Second, as more scholars begin examining Twitter, it is important to have a grounded understanding of the core practices. Third, though Twitter is not universally adopted, Twitter supports an active community with its own set of unique practices that are valuable to examine.

Retweeting is also an important practice to analyze because of the larger issues it raises concerning authorship, attribution, and communicative fidelity. In an environment where conversations are distributed across the network, referents are often lost as messages spread and the messages themselves often shift. What 
participants value and the strategies they use when retweeting reveal salient aspects of the conversations they seek to create on Twitter.

Before examining retweeting as a conversational practice, we begin by describing Twitter and the mechanics of Twitter. We then describe the different data we use, locating retweeting in a broader context. Using these data, we analyze the syntax of retweets, how people retweet, why they retweet, and what they retweet. We then turn to a series of case studies to look more closely at specific conversational practices. In aggregate, this paper serves to highlight the diverse ways by which participants embrace retweeting.

\section{Twitter}

\subsection{Twitter background}

Twitter is a microblogging service that was founded in early 2006 to enable people to share short textual messages - "tweets" - with others in the system. Because the system was originally designed for tweets to be shared via SMS, the maximum length of a tweet is 140 characters. Though the service evolved to include more uses besides SMS, such as web and desktop clients, this limitation persisted, and so was re-narrated as a feature. Twitter's Creative Director Biz Stone argues, "creativity comes from constraint" [16].

Twitter combines elements of social network sites [3] and blogs [13], but with a few notable differences. Like social network sites, profiles are connected through an underlying articulated network, but these connections are directed rather than undirected; participants can link to ("follow") others and see their tweets, but the other user need not reciprocate. Like blogs, participants' Twitter pages show all of their tweets in reverse chronological order, but there is no ability to comment on individual posts. User profiles are minimal and public, but users can make their tweet stream public or protected (a.k.a. private); the default and norm is public.

The central feature of Twitter, which users see when they $\log$ in, is a stream of tweets posted by those that they follow, listed in reverse chronological order. Participants have different strategies for deciding who they follow-some follow thousands, while others follow few; some follow only those that they know personally, while others follow celebrities and strangers that they find interesting.

Although people can interact with Twitter directly through the website, there are many third party applications available, ranging from mobile and desktop Twitter clients to tools that allow participants to track popular topics, who un-follows whom, and how popular different users are. The ecosystem around Twitter is extensive because Twitter makes an API available for developers. For a more detailed guide to Twitter, see [14].

\subsection{Twitter conventions}

Twitter participants are constrained to expressing themselves in 140 characters. As participants embraced the technology and its affordances, a series of conventions emerged that allowed users to add structure to tweets. For example, users developed ways to reference other users, converged on labels to indicate topics, and devised language to propagate messages.

Twitter participants began using the @user syntax to refer to specific users (e.g., @amandapalmer) to address one another. This convention stems from an older IRC practice and serves multiple purposes in Twitter, including directing messages to specific people as though sending the message to them (also known as@replies), and to obliquely reference another user (e.g. "I saw @oprah's show today”).

Honeycutt and Herring point out that the use of @ user is a form of "addressivity" [9], or indicating intended recipients of messages that are posted in an otherwise public forum in order to gain the target person's attention, which is essential for conversation to occur. Honeycutt and Herring [9] also point out the use of @user messages to reference other users. We note here the function of such messages is also attention-seeking; it is a specifically intended to alert the mentioned person that they are being talked about. In the previous paragraph's example, for example, it is unlikely that the tweeter expects a response from Oprah, though they potentially hope for one.

Topics are indicated through the combination of a hashtag (\#) and a keyword. The practice of using keywords to label tweets most likely parallels the use of "tags" to freely categorize web content. Tagging gained visibility with social bookmarking [6], but has expanded to other social media genres, including blogs. The practice of using hashtags may stem from a history among computer programmers of prefacing specialized words with punctuation marks, such as $\$$ and $*$ for variables and pointers, or the \# sign itself for identifying HTML anchor points.

Early Twitter users also began providing links to outside content by including the URL in their tweets. Because URLs are typically long, they take up too many characters. Thus, people started using "URL shorteners" (e.g., http://bit.ly) to generate unique, abbreviated URLs that redirect to the desired website. Sharing links is a central practice in Twitter. 
Retweeting, the focus of this paper, is another emergent practice. Unlike@replies and hashtags, the conventions for retweeting are hugely inconsistent. The prototypical way of retweeting is to copy the message, precede it with RT and address the original author with @. For example:

$$
\begin{aligned}
& \text { A: Hello world! } \\
& \text { B: RT @A: Hello world! }
\end{aligned}
$$

Retweet data is rarely formatted as cleanly as in this example. There is no consistent syntax to indicate a retweet, attribution is inconsistent, the 140-character limitation and other factors prompt users to alter the original message, and adding commentary is prevalent. Furthermore, people use retweet language to reference content from other media and when paraphrasing others' tweets. As a result, the text and meaning of messages often change as they are retweeted and the inconsistent syntax makes it difficult to track the spread of retweets. In tracking and examining retweets, we had to account for these issues.

While these practices were not built into Twitter, Twitter responded to some user convention by building the collectively established syntax into the system. @ user now results in a hyperlink to that user's Twitter page, and a special page exists so users can see all the @ messages mentioning them in a single page; users are also provided with a button to @ reply to any given message which helps track the referent. Hashtags show up in Twitter search and in its "trending topics."

As of this writing, Twitter has announced plans to build retweeting into Twitter's structure as they have done for@replies and \#hashtags, but the functionality has not been released yet. Though some third-party software clients support these practices (e.g. providing buttons to auto-format a retweet), they, like their users, employ several of the syntax conventions we describe here.

\subsection{The construction of a retweet}

There is no universally agreed-upon syntax for retweeting, though the prototypical formulation is ' $\mathrm{RT}$ @ user ABC' where the referenced user is the original author and $\mathrm{ABC}$ is the original tweet's content. We also found the following syntax used to mark retweets: 'RT: @', 'retweeting@', 'retweet @', '(via@)', 'RT (via@)', 'thx@,' 'HT@', 'r@', and ‘0 @'.

Users have different reasons for choosing these different styles. Some default to the style of the thirdparty client they use. Others use different conventions depending on what they wish to achieve (e.g., (a) nav_een: If I paraphrasel remove words I say "via" instead of RT. I'm hopin this is ok Twitettiquette). Each style also has a different convention for locating it in the retweet, which influences some people's practices.
While most of these appear before the content, '(via @ user)' and 'thx user' typically come at the end.

Participants' interpretation of RT and via may also have to do with the ways in which these practices are similar to ones involving earlier media. RT can be seen as analogous to email forwarding - a message is resent to others, verbatim, due to its unique content or insight. This model centers on the original author. In contrast, via is more akin to weblogging practices where knowing who shared the content is key. In such a model, it is common to link to the source and then add content.

Like email chain letters, Twitter users retweet tweets that have already been retweeted by others. Most who retweet give attribution using the @user format. Yet, there does not seem to be a clear standard on how - or whether - to acknowledge all those who came before, just the first, or just the most recent.

Some participants add additional content when they retweet, either before the message or in parentheses or brackets afterwards. Such use of retweeting may be more about the comment with the original text provided for clarity. This parallels 'quoting' in email and on Usenet to provide conversational context [5].

While some conventions have formed, some participants do not understand or choose to reject the conventions of Twitter. For example, there are retweet formulations like 'RT \#y' where ' $y$ ' is a hashtag topic. Participants also use retweet language to quote statements people say verbally or on other social media where there is no origin tweet. The construction of a retweet is further complicated by the ways in which people alter the content of the original tweet.

\section{Data}

To assess retweeting as a practice, we draw on four distinct but complementary data sets. The first two datasets provide quantitative context for understanding retweeting; the second two describe data we use more directly in our analysis and discussion.

\subsection{Random sample of tweets}

The first dataset is a random sample of 720,000 tweets captured at 5-minute intervals from the public timeline over the period 1/26/09-6/13/09 using the Twitter API. This sample includes tweets from 437,708 unique users, but does not include tweets from those with protected accounts. This data set provides valuable insight into the prevalence of a variety of Twitter practices. Using this data, we found that:

-36\% of tweets mention a user in the form '@user'; 86\% of tweetswith@user begin with@user and are presumably a directed @reply 
- $5 \%$ of tweets contain a hashtag (\#) with $41 \%$ of these also containing a URL

- $22 \%$ of tweets include a URL ('http:')

- $3 \%$ of tweets are likely to be retweets in that they contain 'RT', 'retweet' and/or 'via' (88\% include 'RT', $11 \%$ include 'via' and $5 \%$ include 'retweet') ${ }^{1}$

\subsection{Random sample of retweets}

Our second set of data is a random sample of 203,371 retweets captured from the Twitter public timeline using the search API over the period 4/20/09$6 / 13 / 09$. This sample is only from those who have public accounts and includes tweets from 107,116 unique users. This second set of data was captured independently of the first set through explicit queries for retweets of the form 'RT' and 'via'. While other syntax is often used to indicate retweeting and we certainly missed many retweets, these two variants still provide a diverse dataset of retweets. Analyzing these, we found that:

- $18 \%$ of retweets contain a hashtag

- $52 \%$ of retweets contain a URL

- $11 \%$ of retweets contain an encapsulated retweet (RT@user1 RT@user2 ...message..)

-9\% of retweets contain an @reply that refers to the person retweeting the post

Compared to the random sample of tweets, hashtag usage and linking are overrepresented in retweets.

Of retweets containing RT, 5\% were not followed by@user. In some cases, this was because the user didn't use the @ symbol (e.g., 'RT: username'). In other cases, there was a URL but no apparent attribution (e.g., 'RT http://url.com') or 'RT' followed by a quote and attributed to 'Anonymous.'

We also found that $11 \%$ of retweets containing RT included text before the RT; these appear to mostly be commentary on the retweeted content.

Over $9 \%$ of all retweets include a reference to the retweeter's handle. In other words, A retweets B when B's message refers to A. We call these 'ego retweets'.

\subsection{Selected topical stories and threads}

In analyzing retweets, we found that some were part of larger stories or events. As part of a separate project, we used the search API to collect retweets that referenced topically similar phrases from high-profile events (e.g. the Iranian election). We conceptualized these as a "story." We algorithmically analyzed which

\footnotetext{
${ }^{1}$ Our regular expression scripts include variations like 'RT:' and 'retweeting' and exclude words like 'art'.
}

retweets were likely to belong to the same retweet thread. While this program and our story analysis are outside the realm of this paper, we used this tool to capture the retweet case studies used in section 5. We selected examples that highlighted key issues based on our participation in an observation of Twitter.

\subsection{Data on people's practices}

Our final data set consists of qualitative comments on Twitter practices stemming from responses we received to a series of questions on @zephoria's public Twitter account, which has over 12,000 followers:

- "What do you think are the different reasons for why people RT something?" [99 responses]

- "If, when RTing, you alter a tweet to fit under 140 chars, how do you decide what to alter from the original tweet?" [96 responses]

- "What kinds of content are you most likely to retweet? (Why?)" [73 responses]

The responses we received from this convenience sample are not representative of all Twitter users nor do they reflect all possible answers. @zephoria's followers tend to be reflective tech-savvy adults interested in social media, education, and technology. Nevertheless, when asking this relatively homogenous population about their practices, we received very diverse answers. We include them in this paper because they show diversity among even a narrow, biased sample. When we quote specific responses in this paper, we attribute their contributions using the @ user format to reflect the most pervasive retweeting attribution style.

\section{Retweeting practices}

While retweeting conventions have emerged, it has not yet stabilized as a practice. Participants have different beliefs about how retweets are "supposed" to work and this results in varied, and often conflicting, conventions. This is further complicated by third party apps that use different syntax to mark retweets. In this section, we discuss variations in how respondents modify retweets, what content they choose to retweet, and their motivations for doing so.

\subsection{How people retweet}

The increased ambiguity about behavior surrounding retweeting likely comes from the fact that retweeting has more structural complexity than @replies and \#hashtags. The idea of tagging a message with a descriptive keyword or sending a message "to" 
another user via an @reply is fairly straightforward; essentially, information is being added to a message without altering the content of the message. In contrast, and somewhat counterintuitively (since the message is typically copied verbatim), the information content of a retweet is changed. Yet, the constraints surrounding the 140-character limit become pronounced when considering retweeting. Since many users make full use of the 140 characters available, adding "RT @user" to a retweet can require ten or more characters that are not available. As a result, the retweeter must somehow shorten the text of the tweet in order to make it fit. This is even more significant if the retweeter wants to not only rebroadcast another's tweet, but also add commentary.

Since it is not immediately clear how to address the issues presented by retweeting, users have adopted different strategies. Some alter or delete content; others paraphrase. Still others simply do not retweet messages that would necessitate some kind of shortening (e.g., @eslchill: Tweets of 130+ characters are too long to be RTed). There are even those who believe that it is up to the twitterer to leave room for the "RT @user" to be added. This may add a strategic dimension to twittering where if one wants to be retweeted, they must make it easy for others to retweet them.

This section briefly addresses some of the most prominent ways in which people address the limitations in determining how to retweet.

4.1.1. Preservers and adapters. In deciding how to adjust a tweet for retweeting, a significant divide arises between respondents who seek to preserve as much text of a tweet as possible and those who are willing to adapt retweets by removing various parts of the tweet that were, in their opinion, nonessential.

"Preservers" emphasize maintaining the original intent, context, and content (e.g., @DanMerzon: I shorth words, del unnecessary [punctuation,] ... but don't change meaning or attribution.). Among "preservers," there is a continuum from preserving the content to preserving the meaning of the tweet being retweeted (e.g., @danielbeattie: [I change] whatever seems to not alter the original intent.).

Those who fall into the category of "adapters" are willing to remove various parts of the tweet to suit their own purposes. For example, some who use Twitter to share URLs see this as the only essential piece of content and opt to remove some or all of the original tweeter's comment. It is also common-both descriptively and empirically-for users to write their own text that paraphrases the original tweet. Another type of adapter simply truncates the original message to make it fit, regardless of the contextual implications (e.g., @korinuo: I guess is ok to delete the last parts of the message to make it fit and substitute with . .).

In editing, a retweeter can change the intellectual ownership of the substantive content of the message, and retweeters sometimes serve more as "authors" of ideas than "curators" of others' work. Of course, modification is also the basis for how messages get transformed as they are spread across the network, sometimes resulting in a change of meaning as people with different expectations for how one should tweet retweet content.

4.1.2. Shortening retweets through deletion. The most common alteration of a tweet for retweeting is the deletion of individual characters or entire words. This practice is not unique to Twitter; it echoes practices in other media genres (e.g., texting and IM). Since Twitter began with a focus on the 160-character SMS messaging platform, it follows that some Twitter conventions resemble those on that platform. Indeed, shortened retweets resemble "txt spk" [1]. While this approach is interpretable, some users find it objectionable (e.g., @PeterKretzman: best judgmentbut NOT text msg style!).

Another approach to shortening is to 'disemvowel' tweets by removing vowels. A play on the word 'disemboweling,' disemvoweling rose to prominence on the popular BoingBoing blog when the community manager sought to reduce the visibility of offensive content without censoring comments [11]. Content without vowels is more difficult to read, but the words remain interpretable with a little bit of extra cognitive effort. While disemvoweling in retweeting is not a social sanction, the linguistic convention is similar and some tech-savvy participants took cues from this practice in developing their shortening techniques.

An arguably more significant method of shortening a retweet includes removal of entire words. While many users do this, their strategies differ. The goal is typically to remove "extra" or "unnecessary" words (e.g., @pfsorenson: I cut out all the unnecessary words-like (old-fashioned) telegrams: no conjunctions, articles, only critical adjectives, etc.). Respondents reported removing several parts of speech, including prepositions, articles, adjectives, adverbs, but it is worth noting that no respondents suggested removing nouns (except pronouns) or verbs.

4.1.3. Authorship and attribution. As messages are altered, it can be difficult to discern who is being addressed and who is being cited. Ambiguities abound, both with respect to pronoun usage in the content of messages and in conjunction with the attribution protocols surrounding retweeting. For example, when a message is retweeted, the authorship of the message 
changes, adding ambiguity to personal pronouns. Who is the "I" in a retweet? Is it the retweeter, or the retweeted? Consider the following example:

A: I like piña coladas.

B: RT @A: I like piña coladas

B's goal here is ambiguous. Is the reader intended to learn the fact that A likes piña coladas, or is the reader supposed to interpret B as saying "I [too] like piña coladas"? Resolving these ambiguities is a challenge.

As retweets are spread, the use of layered attribution introduces a second set of concerns. Consider the following example:

A: Hawaii is beautiful!

B: RT @A: Hawaii is beautiful!

C: RT @A: Hawaii is beautiful! (via @B)

$A$ reader who sees only $C$ may not know if the original was said by $\mathrm{B}$ and retweeted by $\mathrm{A}$ or vice versa.

A third issue emerges because multiple attributions require additional characters in a constrained environment. Some users believe that it's critical to attribute the chain of authors who passed along the message because this provides context and credit. Some chop the text such as to exclude multiple attributions (e.g., @eileen53 first cut is the string of sources if it's already been RT'ed). However, it is not clear to whom, or to how many people, credit is owed. In some cases, users choose to attribute only the initial author while in other cases, it means attributing the last referent. This latter convention suggests that the appropriate thing to do is to include only one step backwards in the chain of retweets to the most recent "transmitter." Differences in approach can lead to inaccuracies as messages are retweeted. When one interprets attribution as citation, credit may go to the wrong person.

\subsection{Why people retweet}

Retweeting is not a universally adopted practice on Twitter and those who do retweet are not necessarily representative of all types of Twitter users. There are many different incentives for using Twitter [12] and those who are using Twitter for "daily chatter" are less likely to be retweeting than those who are trying to engage in conversations or share information. Yet, among the subpopulation that does retweet, there are diverse motivations for doing so. A non-exhaustive list of motivations based on responses to @zephoria's questions include:

- To amplify or spread tweets to new audiences (e.g., (a)rootwork: RT sees value and amplifies it and (a)lazygal: that which I think the majority of my "followers" haven't seen already)

- To entertain or inform a specific audience, or as an act of curation (e.g., @jmccyoung: to inform or amuse the handful of people who follow me)
- To comment on someone's tweet by retweeting and adding new content, often to begin a conversation (e.g., @anitsirk: to start a conversation about the content of the tweet)

- To make one's presence as a listener visible (e.g., (a)doctorlaura: it shows that one is not just talking, but also listening)

- To publicly agree with someone (e.g., @rzouain: retweets are the 'me too' 2.0 )

- To validate others' thoughts (e.g., @amandapey: because sometimes, someone else just says it better)

- As an act of friendship, loyalty, or homage by drawing attention, sometimes via a retweet request

- To recognize or refer to less popular people or less visible content (e.g., @laurelhart: to support under-recognized people or topics)

- For self-gain, either to gain followers or reciprocity from more visible participants (e.g., @gravity7: to increase own followers, as a favor, possibly for the return favor (from influencer))

- To save tweets for future personal access (e.g., (a)peteaven: so I can find the tweet later by searching on myself, checking my updates)

While some value retweeting, others lament users' selfish motivations (e.g., @earth2marsh: at best retweets altruistically propogate interesting info with credit to originator. At worst it's pandering for social capital" and @argonaut: educated gossiping meets karma whoring). In doing so, they acknowledge that retweeting can be both a productive communicative tool and a selfish act of attention seekers.

\subsection{What people retweet}

What people retweet is also varied, although heavily connected to the reasons for why they retweet. In asking people what they retweeted, some reported favoring retweets of time-sensitive material and breaking news. However, there is disagreement as to what type of time-sensitive material is worth spreading (e.g., @DavidCRoberts: Everything is urgent so only the fun and interesting stuff gets the RT).

Breaking news tends to be retweeted in the form of links to articles in media sources. However, links of many kinds are sent, including material that is of enduring interest to friends or topically relevant to those interested in a particular topic.

One interpretation of Twitter's value derives from the real-time nature of the conversations it supports. Its search and "trending topics" functionality captures public conversations in real time from its entire user population, and this temporality has moved Google to spend more effort considering "real time search" [15]. This may influence what some users choose to retweet. 
4.3.1. Retweeting for others. While some users retweet content of general interest, others are more concerned about the audience to whom they are retweeting (e.g., @viller: most oftenwhen I see a link from someone that I think is interesting to ppl who follow me ie. close(ish) ties). In choosing what to retweet, these participants often think explicitly about who follows their tweets. Though Twitter users can access a list of who follows them, this is not necessarily their actual audience. As such, participants must contend with an imagined audience, just as they do when using other social media [2]. This is only further complicated when people must account for the overlap in their followers and that of those who they follow. Granovetter [7] points out that, despite modern fascination with the idea of small worlds, the point behind Milgram's experiment was that pairs of people were surprised when they learned they share a contact, remarking on what a small world it is. Granovetter further points out the cognitive effort required to keep track of not only alters, not to mention the ties between that set of alters, is immense.

When participants choose to retweet messages they see from followers, there may be an overlap between their potential audience and the potential audience of the originator, but the retweeter is unlikely to know what that overlap might be.

Compounding this, potential followers and friends are not necessarily the best method of measuring who is paying attention to whom. Huberman, et al [10] point out that users who@reply one another form a more significant network of ties. Given the inherent difficulty in following these messages, since they may or may not be visible to other parties, keeping track of who truly pays attention to whom is hopeless.

Nevertheless, respondents consistently sought to account for their audience when choosing what to retweet (e.g., @simoncolumbus: links i think most of my followers don't know and @viller: I sometimes hold back if I think that many of those who follow me also follow the same person I am RTing). As such, the intended audience plays a role in shaping what some people retweet.

4.3.2. Retweeting for social action. Many retweets appear to encourage different types of "social action." Some have serious requests in them, such as calls to protest or donate. For example, some users retweeted @ suzymiles' tweet "is going to the Arctic to raise 10k for the Willow Foundation http://URL (pls RT/donate to help)." These calls can be quite effective. Hundreds of users retweeted the message "RT@StopAhmadi Bring down Khomeini's website” with a link to his site; shortly later, the site faltered.
Other social action retweets involve demonstration of collective group identity-making. For example, in the first 24 hours after the shooting of George Tiller, nearly 400 users retweeted the message, "Pro-life leaders condemn murder of abortionist", and many of these tweets contained the text, "RETWEET THIS UNTIL IT TRENDS". That is, the retweeters were attempting to make the topic so popular that it would appear on Twitter's "trending topics" page and thus be broadcast to a wide number of twitter users who might otherwise not encounter it. This is an example of Twitter use attempts to manipulate the Twitter system itself in order to effect social action.

Another type of social action stems from the power of Twitter as a "crowdsourcing" mechanism. Users retweet messages that request help to leverage the knowledge, skills, and contacts of their followers (e.g., (a)billsimmon: crowdsourcing answers to questions and group problem solving deserve RTs).

Retweeting for social action is most successful when the retweeter has a large network and occupies structural holes, or gaps in network connectivity between different communities [4]. In order to be able to spread information to new people, the individual must be connected to those to whom the source of the information is not connected already. Additionally, celebrities and other highly followed users are in a particularly good position to broadcast content for social action.

Social action retweets are purposeful in nature and thus what people retweet is often tied to why they retweet. In short, the content people retweet is inextricably tied to the goals they have related to selfimage and self-promotion, supporting conversation and building community.

\section{Example retweeting conversations}

Retweets can knit together tweets and provide a valuable conversational infrastructure. Whether participants are actively commenting or simply acknowledging that they're listening, they're placing themselves inside a conversation. Even when they are simply trying to spread a tweet to a broader audience, they are bringing people into a conversation.

The types of conversations that emerge on Twitter through retweeting are as diverse as the conventions upon which they reside. Like those conversations that take place through @replies [9], some retweet conversations are small and local while others prompt a huge thread. Additionally, retweeting is sometimes used to take what could be an @reply conversation and bring in broader audiences. Of course, not all retweeted conversations retain their original meaning. This 
section contains four sets of case studies that reveal some of the dynamics we have mapped out thus far.

\subsection{The flow of a retweet thread}

When a topic captures the attention of a group of people, they may want to share the topic with others as well as offer their own commentary. However, as the topic is shared and reshared through retweeting, the conversation can morph in several interesting and unpredictable ways. To describe the flow of retweets, this section uses one case study based on two tweets by the first author (@zephoria) posted ten days apart:

1) qotd: "Facebook is for old people!" (exclaimed by $14 y o$ when I asked her why she preferred MS over FB; complete $w /$ look of horror)

2) new blog post "Is Facebook for old people?" is based on interviews $w /$ teens in Atlanta last week http://bit.ly/v0aPS

These two posts were retweeted or referenced in others' tweets approximately 130 times in a little over two weeks, and throughout the process were changed several times along the way, with added comments, deletions, and so on.

The commentary often consisted of a brief note placed at the beginning of the retweet just before "RT", usually endorsement (e.g., "(fascinating)") or brief summary (e.g., "class matters"). When comments were brief, such as these, they most often appeared at the very beginning, but longer comments typically followed, rather than preceded, the retweet:

@ptanthos: RT @zephoria: newblog post"Is Facebook for old people?" http://bit.ly/v0aPS. What

$r$ others seeing? Are adults causing kids to flee?

In this retweet, the additional description about the Atlanta field site is removed completely in order to make room for the retweeter's commentary. Such comments might at once demonstrate retweeters' access to interesting content, as well as their desire to stimulate discussion among their own followers.

Not all retweets stemming from this particular case study are formally marked as retweets. This is particularly true when messages are paraphrased rather than directly quoted. Yet, such messages can still serve the function of a retweet even if they are not marked as such. Consider:

(a) brianeisley: Librarians interested in Web 2.0 should pay close attention to @zephoria. Case in point: http://bit.ly/v0aPS.

Two elements make this likely to be a retweet: 1) @brianeisley follows@zephoria; 2)@brianeisley's tweet uses the same shortened URL as in @zephoria's original post. The latter point is notable because URLs that are shortened have their own signature; the reuse of a shortened URL suggests a reference. While we found such examples in this case study, accounting for these examples in broader datasets is challenging.

To illustrate the multiplicity of ways a retweet can be constructed and the differences in how attribution and comment propagation, consider one retweet thread:

1)@mStonerblog:RT@zephoria: newblog post "Is Facebook for old people?" based on interviews w/teens in Atlanta http://bit.ly/v0aPS. Always insightful!

2)@jtoddb:RT@mStonerblogRT @zephoria:new blog post "Is Facebook for old people?" based on interviews w/ teens in Atlanta http://bit.ly/v0aPS.

3)@sparepixel: RT@mStonerblog:new blog post "Is Facebook for old people?" based on interviews w/ teens in Atlanta http://bit.ly/v0aPS. Always insightful!

4)@AndreaJarrell:Via@mStonerblog:RT @zephoria: new blog post "Is Facebook for old people?" socioecon \& race are most interesting here http://bit.ly/v0aPS.

The first retweet copies the post verbatim, includes the "RT@user" convention, and includes a brief comment at the end, following the link. The second is a retweetof-a-retweet, and the retweeter has chosen to keep the names of both the previous retweeter and the original author, but has dropped the first's commentary. The third is actually mirrors the first's content but, in doing so, makes it clear who the author of the commentary is.

The fourth example is slightly more complex. It also references the first, using both the 'RT' and 'via' conventions, perhaps to distinguish the original retweet and who sent it along. Yet, in this example, the content has morphed in ways that reflect neither the original post nor the referenced retweet. Rather, (a)AndreaJarrell is putting her own spin on the story but not distinguishing it from the original or the referenced retweet. In this way, the authorship of the content becomes ambiguous.

In another retweet, @mStonerblog's tweet is passed out without the original reference to @zephoria:

@sparepixel:RT@mStonerblog:new blog post"Is

Facebook for old people?" based on interviews w/

teens in Atlanta http://bit.ly/v0aPS. Always

insightful!

In this example, the original author is completely removed and the most recent transmitter is RT'ed. Note that this tweet is structurally identical to the first one from@mstonerblog above; if in the former, it was interpreted that @zephoria was the blog post author, then the same is true here, and a reader might interpret @ mstonerblog to be the author of the blog post that is referenced. The retweeter here likely meant no harm, but this example illustrates how different models of 
attribution (credit the previous transmitter versus credit the original author) can lead to serious confusion about who is responsible for what. Retweeting attribution adds a new twist to the death of the author.

\subsection{Requesting a retweet}

Retweeting can be a political act, especially amongst those who wish to get their voices heard. It is not unusual for users to ask for their messages to be retweeted. Indeed, some of their followers may oblige their requests. While some may retweet altruistically, there are plenty who seek attention from the person they retweet as well as those who hope that doing so will be reciprocated in the future. What becomes clear in this dynamic is that visibility and status matter.

In response to the post-election street uprisings in Iran, @zaibatsu posted: “Citizen journalist media Pls $R T$ this video channel http://bit.ly/Gae8i". In the hour that followed, over three-dozen users retweeted his message. Not all who did follow him directly and many of the retweets contain embedded retweets. This suggests that the message is reaching new and broader audiences. Examining the Twitter streams of those who retweeted reveals that this message is part of a larger conversation on the Iranian election. Many, but not all, who retweeted this message were tweeting regularly about the Iranian election. The primary contribution of this tweet is the link provided, a link to amateur videos of street protests in Iran. It is clear that @zaibatsu wishes to get this information out, in part to get people talking about the Iranian elections. But not all who retweet his message are engaged in the election in the same way. Thus, the tweet and its subsequent retweets simultaneously contribute to a broader conversation and create a conversation around the link itself.

\subsection{Ego retweets}

Ego retweets are when people retweet messages that refer to them. Some see this as "narcissistic" or "self-serving," while others see it as a way of giving credit to and appreciating the person talking about them. For example, @the1secondfilm suggests that retweeting references to oneself is "Sort of a shout out to a shout out." Consider this example:

@LoriMoreno: I'm glad that You Smiled Colin! RT

@EditorColin@LoriMoreno I looked at and smiled.

Thanks for making that happen

At one level, @LoriMoreno is responding to the tweet. Yet, if this was all she wished to do, she could have @replied directly to @EditorColin. By retweeting, she brings her audience into a conversation, helping them understand the context of what is being discussed before adding her own commentary.
Ego retweeting is also a way publicly appreciate someone else's attention, especially when the original author has more visibility than the person referenced. For example, after @Themelis_Cuiper included (a)StephenWinfield in his \#followfriday list of who to follow, @StephenWinfield retweeted the list and added: "Thanks 4 the \#followfriday tweets". In doing so, he acknowledged@Themelis_Cuiper's status and visibility while also marking his appreciation.

Marketers also use ego retweet when consumers mention positive or interesting things about their brand. As such, their Twitter stream includes the voices of consumers validating them. For example, @southwestair tweets messages like "RT@Jaunted: Penguins on an @southwestair plane!" This can backfire. While@Jaunted is following@southwestair and presumably welcomes the attention, not all who find their messages retweeted by brands appreciate this; many marketers wish to be in conversation with their consumers, not all consumers are looking to be in conversation with marketers.

\subsection{The broken telephone effect}

Not all retweets are an accurate portrayal of the original message. When people edit content to retweet, they may alter the meaning of the original. Even when the content was not altered, taking a tweet out of context can give it a life of its own. Consider these two abbreviated tweets:

1)@eszter: store clerk: "My boyfriend broke up with me w/text messages..." Ouch!

2) (a follower): RT@eszter My boyfriend broke up with me w/text messages..

In shortening the message, @eszter's follower shared what she believed to be the most interesting part of the tweet, but she changed the meaning in the process. Concerned at how others might interpret this, @eszter contacted her follower who deleted the retweet and posted a new tweet apologizing to @eszter and blaming the technology by noting "Darn 160 characters and on my phone." While this case may have been accidental, such incidents regularly occur.

When altered retweets spread virally, conversations can become gossip. Consider these abbreviated tweets:

1)@sstacinator: Al Green died? Auto accident? WTF? ... TRUE OR NOT TRUE?...

2)@aplusk: Have you heard that Al Green died in a auto wreck a little while ago? SAD... (via @sstacinator) is this true?

3)@emeraldjane: RT @aplusk: Have you heard that Al Green died in a auto wreck a little while ago? $S A D \ldots$ 
As@aplusk's message was retweeted, his question was dropped and the message became fact. Further, @aplusk became the source of this story. Yet, Al Green had not died (although a different Al Greenz did); when@aplusk posted a correction, it was barely retweeted.

These cases highlight how misinformation can spread through Twitter, either through alteration or misinterpretation. They also show how conversations in Twitter are not necessarily coherent. Participants do not all hear the same messages or share the same social context. In this way, conversations on Twitter can sometimes take the form of a glorified game of "Broken Telephone" as individuals whisper what they remember to their neighbor and the message is corrupted as it spreads.

\section{Retweets as conversational practice}

The structure of conversations varies, even in groups that are bounded in space, time and participant group. Conversations in bounded groups derive order from turn taking and reference to previous statements (see [8]), but when the conversation is distributed across a non-cohesive network in which the recipients of each message change depending on the sender, these conversational structures are missing. The result is that, rather than participating in an ordered exchange of interactions, people instead loosely inhabit a multiplicity of conversational contexts at once.

In this paper, we have described several variations in the practice of retweeting messages on Twitter and the ways in which varying styles lead to ambiguity in and around authorship, attribution, and conversational fidelity, especially as the content of messages morph as they are passed along.

Though the 140-character format is a constraint, it need not be seen as a limitation; while participants often shorten and otherwise modify tweets to fit into 140 characters, this characteristic of Twitter can also be seen as an advantage. The brevity of messages allows them to be produced, consumed, and shared without a significant amount of effort, allowing a fastpaced conversational environment to emerge. The varied approaches users take in addressing constraints reveal what they value in specific messages and in Twitter as a conversational environment. Participants' social and informational goals vary, and accordingly, so do their retweeting practices. Regardless of why users embrace retweeting, through broadcasting messages, they become part of a broader conversation.

\section{Acknowledgements}

We would like to thank Lucy Mendel for helping collect data and Sarita Yardi for providing feedback.

\section{References}

[1] Baron, N. and R. Ling. Text Messaging and IM: Linguistic Comparisons of American College Data. Journal of Language and Social Psychology. 26: 291-298. (2007).

[2] boyd, d. Taken Out of Context: American Teen Sociality in Networked Publics. PhD Dissertation, University of California-Berkeley, 2008.

[3] boyd, d. and N. Ellison. Social Network Sites: Definition, History, and Scholarship. Journal of ComputerMediated Communication 13, 1 (2007), article 11.

[4] Burt, R. The Network Structure of Social Capital. In R. Sutton and B. Staw, Research in Organizational Behavior. Vol. 22. JAI Press (2000).

[5] Severinson Eklundh, K. and C. Macdonald. The Use of Quoting To Preserve Context in Electronic Mail Dialogues. IEEE Transactions on Professional Communication 37 (4) (1994), 197-202.

[6] Golder, S. and B.A. Huberman. Usage Patterns of Collaborative Tagging Systems. Journal of Information Science 32, 2 (2006), 198-208.

[7] Granovetter, M. Ignorance, Knowledge and Outcomes in a Small World. Science. 301: 773-774. (2003).

[8] Herring, S. Interactional Coherence in CMC. Journal of Computer-Mediated Communication. 4, 4 (1999).

[9] Honeycutt, C. and S. Herring. Beyond Microblogging: Conversation and Collaboration in Twitter. Proc $42^{\text {nd }}$ HICSS, IEEE Press (2009).

[10] Huberman, B., D. Romero and F. Wu. Social Networks that Matter: Twitter Under the Microscope. First Monday. 14(1). (2009).

[11] Jardin, X. Online Communities Rot Without Daily Tending By Human Hands. Edge World Question Center. 2008. http://www.edge.org/ q2008/q08_7.html\#jardin

[12] Java, A., X. Song, T. Finn, \& B. Tseng. Why we Twitter: Understanding microblogging usage and communities. Proc. Joint 9th WEBKDD and 1st SNA-KDD Workshop, ACM Press (2007).

[13] Marlow, C. The Structural Determinants of Media Contagion. PhD Dissertation, MIT Media Lab, 2005.

[14] O'Reilly, T. and S. Milstein. The Twitter Book, O'Reilly, Sebastapol CA, 2009.

[15] Stross, R. Hey Just a Minute (or why Google isn't Twitter). New York Times. June 13, 2009.

[16] Zinko, C. What is Biz Stone doing? San Francisco Chronicle, K-1, April 5, 2009. 